\title{
Insights on left ventricular and valvular mechanisms of recurrent ischemic mitral regurgitation after restrictive annuloplasty and coronary artery bypass grafting
}

Sandro Gelsomino, MD, ${ }^{\text {a }}$ Roberto Lorusso, MD, PhD, ${ }^{b}$ Sabina Caciolli, MD, ${ }^{a}$ Irene Capecchi, MD, ${ }^{\text {a }}$ Carlo Rostagno, MD, ${ }^{\text {a }}$ Marco Chioccioli, MD, ${ }^{a}$ Giuseppe De Cicco, MD, ${ }^{b}$ Giuseppe Billè, MD, ${ }^{a}$ Pierluigi Stefàno, MD, ${ }^{a}$ and

Gian Franco Gensini, MD

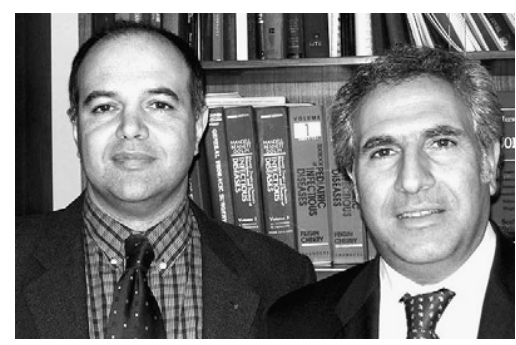

S. Gelsomino (left), R. Lorusso (right)
From the Experimental Surgery Unit, Cardiac Surgery, Department of Heart and Vessels, ${ }^{\text {a }}$ Careggi Hospital, Florence, Italy, and Cardiac Surgery, ${ }^{b}$ Civic Hospital, Brescia, Italy.

Received for publication Jan 12, 2008; revisions received Feb 21, 2008; accepted for publication March 21, 2008.

Address for reprints: Sandro Gelsomino, MD, Experimental Surgery Unit, Careggi Hospital, Viale Morgagni 85, 50134, Florence, Italy (E-mail: sandro.gelsomino@ libero.it).

J Thorac Cardiovasc Surg 2008;136:507-18 0022-5223/\$34.00

Copyright (C) 2008 by The American Association for Thoracic Surgery

doi:10.1016/j.jtcvs.2008.03.027
Background: We investigated leaflet and subvalvular configurations to identify mechanisms leading to recurrent mitral regurgitation after combined undersized mitral annuloplasty and coronary artery bypass and to preoperatively recognize patients who are unlikely to benefit from this approach.

Methods: Among 261 subjects with chronic ischemic mitral regurgitation undergoing undersized annuloplasty and coronary bypass surgery at one institution between September 2001 and September 2007, 31 were excluded: 4 had intraoperative annuloplasty failure, 12 showed residual regurgitation, and 15 had incomplete echocardiograms available. The study population consisted of 230 patients who were divided into 2 groups: patients without (group 1, $\mathrm{n}=176$ ) or with (group $2, \mathrm{n}=54)$ late recurrent mitral regurgitation. Fifty healthy subjects were used as control subjects. Serial echocardiographic analysis was performed preoperatively, at discharge, and at follow-up appointments (early: median, 6 months [interquartile range, 5-6 months; late: median, 33 months [interquartile range, 17-51 months]).

Results: Subjects with late regurgitation had preoperatively more symmetric tethering $(P<.001)$, more accentuated anterior mitral leaflet tethering $(P<.001)$, and more restricted anterior leaflet excursion $(P=.003)$ than patients in group 1. Postoperatively, tethering of the posterior leaflet increased $(P<.001)$ and was predominant in both groups, whereas tethering of the anterior leaflet was reduced at discharge ( $P=.01$ and $P=.03$, respectively), remaining constant afterward. Multivariable analysis showed an anterior tethering angle of $39.5^{\circ}$ or greater $(P<.001)$, an anterior/posterior tethering angle ratio of 0.76 or greater $(P<.001)$, an anterior leaflet excursion angle of $35^{\circ}$ or less $(P=.001)$, and a coaptation height of $11 \mathrm{~mm}$ or greater $(P=.04)$ to be predictors of recurrent mitral regurgitation.

Conclusions: Preoperative symmetric tethering with anterior mitral leaflet predominance was strongly associated with recurrence of mitral regurgitation. Measures of leaflet tethering resulted in fundamental findings to identify ischemic patients who can really benefit from restrictive annuloplasty. Further larger studies are necessary to confirm our results.

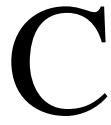
hronic ischemic mitral regurgitation (CIMR) results from a variable combination of infarction-induced subvalvular remodeling with subsequent leaflet tethering and annulus dilatation/flattening. ${ }^{1,2}$ An established therapeutic approach to relieve CIMR in association with coronary artery bypass grafting (CABG) is an undersized mitral ring annuloplasty (UMRA), which, by reducing the septal-lateral dimensions and the valve area, brings both mitral leaflets into apposition. ${ }^{3}$ Nonetheless, after encouraging initial results, ${ }^{4}$ different studies have revealed a significant proportion of patients with recurrent mitral regurgitation (MR) during 


\author{
Abbreviations and Acronyms \\ $\mathrm{AML}=$ anterior mitral leaflet \\ $\mathrm{APM}=$ anterior papillary muscle \\ $\mathrm{CABG}=$ coronary artery bypass grafting \\ $\mathrm{CI}=$ confidence interval \\ CIMR $=$ chronic ischemic mitral regurgitation \\ $\mathrm{CL}=$ coaptation length \\ ERO = effective regurgitant orifice \\ IQR = interquartile range \\ MR = mitral regurgitation \\ $\mathrm{PM}=$ papillary muscle \\ PML = posterior mitral leaflet \\ PPM = posterior papillary muscle \\ $\mathrm{RV} \quad=$ regurgitant volume \\ UMRA = undersized mitral ring annuloplasty \\ WMSI $=$ wall motion score index
}

follow-up, ${ }^{5-7}$ which has been mainly related to continued left ventricular remodeling and persistent leaflet tethering. ${ }^{7,8}$ In our previous experience, ${ }^{9} 72 \%$ of patients undergoing combined CABG and UMRA showed recurrent MR at 5-year follow-up. In addition, left ventricular reverse remodeling was observed only in $44.2 \%$ of the study population, with $10.3 \%$ of patients showing further left ventricular dilatation. In the present study we investigated leaflet and subvalvular configurations before and after surgical annuloplasty to identify mechanisms leading to recurrent MR. Furthermore, we attempted to determine whether preoperative tethering patterns and subvalvular configurations can accurately predict unsuccessful ischemic valve repair.

\section{Materials and Methods \\ Patient Population}

Among 261 subjects with CIMR undergoing combined CABG and UMRA in our institution (Cardiac Surgery, Careggi Hospital, Florence, Italy) between September 2001 and September 2007, 31 were excluded: 4 had intraoperative annuloplasty failure, 12 had residual MR ( $\geq 2+$ at discharge), and 15 had incomplete echocardiograms available. Definitions and inclusion criteria are as previously reported. ${ }^{9}$ Therefore the study population consisted of 230 patients who were divided into 2 groups: patients without (group 1, $\mathrm{n}=$ 176 ) or with (group 2, n $=54$ ) late recurrent MR (insufficiency $\geq 2+$ at late appointment in patients with no/trivial MR at discharge). Fifty healthy subjects with no history of cardiovascular disease and normal Doppler echocardiographic examination results who had sex distributions, ages, and average body surface areas similar to those of study patients were used as control subjects. Patient profiles are summarized in Table 1. According to the World Medical Association guidelines concerning ethical principles for medical research involving human subjects, ${ }^{10}$ the study was approved by the institutional ethics board. Furthermore, all patients provided informed consent.

\section{Surgical Intervention}

All patients underwent associated CABG. For the purpose of this study, complete revascularization was accomplished when at least 1 graft was placed distal to an approximately $50 \%$ diameter narrowing in each of the 3 major vascular systems in which arterial narrowing of this severity was noted in a vessel of $1.5 \mathrm{~mm}$ or greater of diameter. It was not considered necessary to bypass all obstructed diagonal branches of the anterior descending or marginal branches of the circumflex coronary arteries for a classification of complete revascularization. Following this definition, $100 \%$ of patients underwent complete revascularization. The ring size was determined by means of standard measurements of the intertrigonal distance and anterior leaflet height. A downsizing by 2 ring sizes was performed in all patients. After cardiopulmonary bypass, transesophageal echocardiographic analysis was performed to assess residual MR.

\section{Echocardiographic Analysis}

Serial transthoracic echocardiographic analysis was performed preoperatively, at discharge, and at follow-up appointments (follow-up $100 \%$ complete). Median early follow-up was 6 months (interquartile range [IQR], 5-6 months), and median late follow-up was 33 months (IQR, 17-51 months). Echocardiographic examinations were carried out by 2 experienced echocardiographers (S.C. and M.C.) and stored on a magneto-optical disc for offline analysis. Measurements were made offline by 2 cardiologists (I.C. and C.R.) blinded to the severity of MR and to the time when the images were made. Measurements and calculations were carried out as previously reported. ${ }^{9}$ The reliability of echocardiographic measurements was assessed by calculating interobserver and intraobserver interval of agreements of main direct measures used in this study in a different group of 20 subjects (10 with MR, Appendix 1). ${ }^{11}$

\section{Quantification of MR}

The following quantitative measurements were simultaneously used to grade the severity of MR, and final results were averages of measured values. ${ }^{12,13}$

Quantitative Doppler scanning. Mitral and aortic stroke volumes were calculated, and regurgitant volume (RV) was the difference between these 2 stroke volumes. The effective regurgitant orifice (ERO) area was the ratio of RV to regurgitant time velocity integral.

Proximal isovelocity surface area. Proximal isovelocity surface area was used to analyze the proximal flow convergence, and ERO was the ratio of regurgitant flow to regurgitant velocity. RV was the product of ERO by regurgitant time velocity integral. For each measurement, a minimum of 3 cardiac cycles were averaged. The severity of MR was graded on a scale of 1 to 4 according to American Society of Echocardiography guidelines. ${ }^{12}$ In patients with no/trivial $\mathrm{MR}$, as determined by means of color Doppler scanning, RV and regurgitant fraction were used as calculated, and ERO was assumed as null. Mitral annular areas were obtained from mitral annular dimensions in apical long-axis, 4-chamber, and 2-chamber views by using an ellipsoid assumption ${ }^{14}$ and indexed by body surface area.

\section{Papillary Muscle Displacement}

The displacement of papillary muscles (PMs) was quantified as distances from well-defined anatomic landmarks at early and end-systole. ${ }^{15}$ The geometric chord defined by septal insertions 
TABLE 1. Patient profile

\begin{tabular}{|c|c|c|c|c|}
\hline & All, $n=230$ & Group1 (MR-), n = 176 & Group $2(M R+), n=54$ & $P$ value \\
\hline Age, y & $67 \pm 7$ & $68 \pm 8$ & $66 \pm 7$ & .87 \\
\hline Sex, M/F & $142 / 88(63.1 / 36.9)$ & $106 / 70(60.2 / 39.8)$ & $36 / 18(66.6 / 33.4)$ & .07 \\
\hline NYHA class & $3(3-4)$ & $3(3-4)$ & $3(3-5)$ & .66 \\
\hline CCS angina class & $2(1-3)$ & $2(1-3)$ & $2(1-3)$ & $>.9$ \\
\hline \multicolumn{5}{|l|}{ EuroSCORE } \\
\hline Additive & $8.1(5-9)$ & $8.0(5-9)$ & $8.2(5-8)$ & .8 \\
\hline Logistic & $15(10-18)$ & $14.8(11-18)$ & $15.1(10-18)$ & \\
\hline Hypertension & $89(38.7)$ & $67(38.1)$ & $22(40.7)$ & .6 \\
\hline Diabetes & $66(28.6)$ & $50(28.4)$ & $16(29.6)$ & .81 \\
\hline COPD & $31(12.7)$ & $23(13.0)$ & 8 (12.5) & .9 \\
\hline Chronic renal disease & $35(15.2)$ & $26(14.8)$ & $9(16.6)$ & .77 \\
\hline Cerebral vascular disease & $25(10.8)$ & $18(10.2)$ & $7(12.0)$ & .63 \\
\hline Peripheral vascular disease & $16(6.9)$ & $12(6.8)$ & $4(7.4)$ & .83 \\
\hline Family history & $114(49.5)$ & $85(48.3)$ & $29(53.7)$ & .57 \\
\hline \multicolumn{5}{|l|}{ Myocardial infarction } \\
\hline$>3 \mathrm{mo}$ & $175(76.1)$ & $132(75.0)$ & $43(79.6)$ & \\
\hline$\leq 3 \mathrm{mo}$ & $55(23.9)$ & $44(25.0)$ & $11(20.4)$ & \\
\hline Inferior/posterior & $121(52.6)$ & $98(55.7)$ & $23(42.5)$ & .8 \\
\hline Anterior/septal & $20(8.7)$ & $14(7.9)$ & $6(11.1)$ & \\
\hline Lateral & $22(9.6)$ & $15(8.6)$ & 7 (12.9) & \\
\hline Combined & $67(29.1)$ & $49(27.8)$ & $18(33.5)$ & \\
\hline Coronary vessel disease & $2(2-3)$ & $2(2-3)$ & $2(2-3)$ & \\
\hline 1 & $5(2.2)$ & $3(1.8)$ & $2(3.8)$ & \\
\hline 2 & 103(44.7) & $77(43.7)$ & $26(48.1)$ & .66 \\
\hline$\geq 3$ & $122(53.1)$ & $96(54.5)$ & $26(48.1)$ & \\
\hline Left main & $43(18.6)$ & $32(18.1)$ & $11(20.3)$ & \\
\hline \multicolumn{5}{|l|}{ Medications } \\
\hline Angiotensin-converting enzyme inhibitors & $186(80.8)$ & $141(80.1)$ & $45(83.3)$ & \\
\hline$\beta$-Adrenergic blockers & $124(53.9)$ & $96(54.5)$ & $28(51.8)$ & \\
\hline Long-acting nitrates & $105(45.6)$ & $82(46.6)$ & $23(42.5)$ & .71 \\
\hline Diuretics & $195(84.7)$ & $150(85.2)$ & 45 (83.3) & \\
\hline Digitalis & $49(21.3)$ & $38(21.5)$ & $11(20.3)$ & \\
\hline Calcium antagonists & $35(15.2)$ & $26(14.7)$ & $9(16.6)$ & \\
\hline Preoperative IABP & $14(6.1)$ & $11(6.2)$ & $3(5.5)$ & 6 \\
\hline \multicolumn{5}{|l|}{ Operations } \\
\hline Antegrade cardioplegia & $208(90.4)$ & $158(89.7)$ & $50(92.5)$ & 6 \\
\hline Antegrade and retrograde cardioplegia & $22(9.6)$ & $18(10.3)$ & $4(7.5)$ & .16 \\
\hline CPB time (min) & $108(95-120)$ & $105(94-118)$ & $110(100-126)$ & .09 \\
\hline CCL time (min) & $90(70-98)$ & $81(66-90)$ & $95(70-101)$ & .06 \\
\hline \multicolumn{5}{|l|}{ Surgical approach } \\
\hline Left atriotomy & $192(83.4)$ & $146(82.9)$ & $46(85.2)$ & .8 \\
\hline Transseptal & $21(9.2)$ & $17(9.7)$ & $4(7.4)$ & .23 \\
\hline Transseptal extended to LA roof & $17(7.4)$ & $13(7.4)$ & $4(7.4)$ & $>.9$ \\
\hline \multicolumn{5}{|l|}{ Mitral ring } \\
\hline Carpentier Classic ${ }^{*}$ & $123(53.4)$ & $93(52.8)$ & $30(55.5)$ & .7 \\
\hline Physio* & $107(46.6)$ & $83(47.2)$ & $24(44.5)$ & .51 \\
\hline \multicolumn{5}{|l|}{ Ring size } \\
\hline $24 \mathrm{~mm}$ & $28(26-30)$ & $28(26-30)$ & $28(26-30)$ & $>.9$ \\
\hline $26 \mathrm{~mm}$ & $12(5.2)$ & $9(5.1)$ & $3(5.5)$ & \\
\hline $28 \mathrm{~mm}$ & $87(37.8)$ & $68(38.6)$ & $19(35.3)$ & \\
\hline $30 \mathrm{~mm}$ & $88(38.3)$ & $67(38.1)$ & $21(38.8)$ & \\
\hline CABG & $43(18.7)$ & $32(18.2)$ & $11(20.4)$ & \\
\hline Anastomoses/patient & $2(2-3)$ & $2(2-3)$ & $2(2-3)$ & $>.9$ \\
\hline Arterial grafts/patient & $1(1-2)$ & $1(1-2)$ & $1(1-2)$ & $>.9$ \\
\hline
\end{tabular}

Continuous variables are presented as means \pm standard deviation, and discrete variables are presented as percentages. Nonparametric variables are presented as medians (interquartile range). MR, Mitral regurgitation; NYHA, New York Heart Association; CCS, Canadian Cardiovascular Society; COPD, chronic obstructive pulmonary disease; $I A B P$, intra-aortic balloon pump; $C P B$, cardiopulmonary bypass; $C C L$, aortic crossclamp; $L A$, left atrial; $C A B G$, coronary artery bypass grafting. *Edwards LifeSciences, Irvine, Calif. 

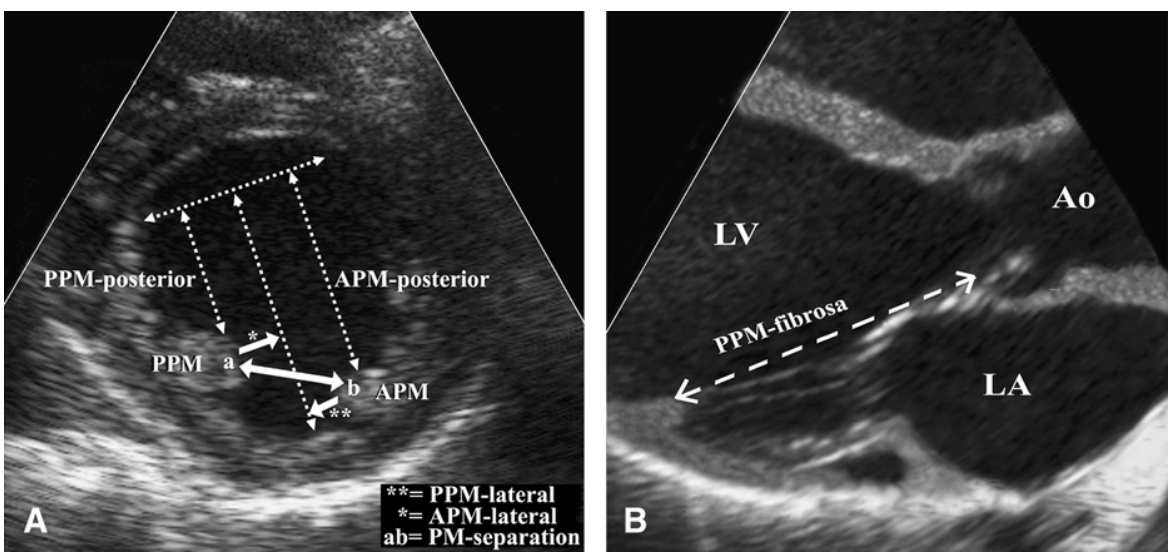

Figure 1. A, Papillary muscle displacement measured in the parasternal shortaxis view. B, posterior papillary muscle-fibrosa measured in the long-axis view (see text). PPM, Posterior papillary muscle; $A P M$, anterior papillary muscle; $P M$, papillary muscle; $L V$, left ventricle; $A o$, aorta; $L A$, left atrium.

and the midseptal perpendicular line were used as references in the parasternal short-axis view (Figure 1, A). Lateral and posterior displacements of anterior papillary muscles (APMs) and posterior papillary muscle (PPMs) were measured as distances from these fixed references. Separation between PMs was directly measured. In the long-axis view the apical displacement of the PPM was measured as the distance between the PM head and the fixed intervalvular fibrosa (annular-papillary distance; Figure $1, B)$. The wall motion score indexes (WMSIs) of the basal, midposterior, and inferior segments for the PPM and the basal, midlateral, and anterior segments for the APM were also calculated. $^{15}$

\section{Mitral Leaflet Tethering}

Mitral leaflet tethering in midsystole was assessed in the parasternal long-axis view (Figure 2). The mitral valve tenting area was calculated as the area enclosed between the annular line and the mitral valve leaflets. Posterior and apical displacement of the coaptation was measured as coaptation distance and coaptation height, respectively, whereas coaptation length (CL) was directly measured. The anterior mitral leaflet (AML) tethering angle, the AML excursion angle and distance, the posterior mitral leaflet (PML) tethering angle, the PML excursion angle and distance, and the bending angle were obtained as explained in Appendix 2. Tethering patterns were defined according to Agricola and coworkers. ${ }^{15}$ The anterior/posterior
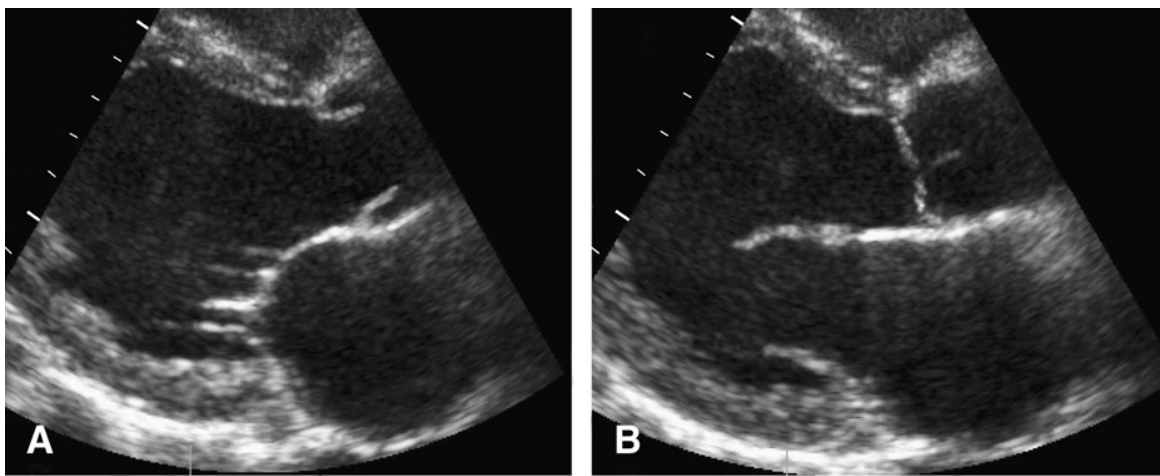

a

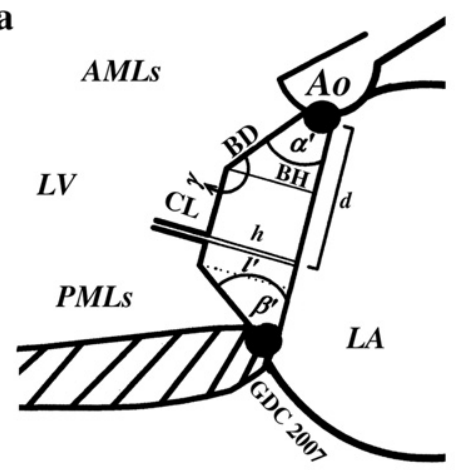

b

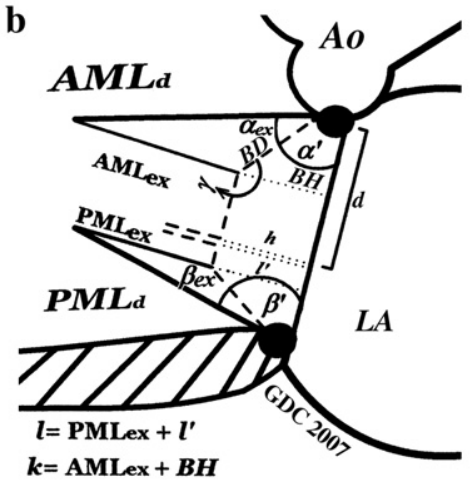

Figure 2. A, Method to quantify mitral leaflet tethering in the parasternal long-axis view in midsystole. AML, Anterior mitral leaflet; $L V$, left ventricle; $P M L$, posterior mitral leaflet; Ao, aorta; $L A$, left atrium; $C L$, coaptation length; $d$, coaptation distance; $h$, coaptation height; $\alpha^{\prime}$, anterior mitral leaflet tethering angle; $\gamma$, bending angle; $\beta^{\prime}$, posterior mitral leaflet tethering angle. $B$, Diastolic parasternal long-axis view. $A M L$, Anterior mitral leaflet; $P M L$, posterior mitral leaflet; $A o$, aorta; $L A$, left atrium; $\alpha_{E X}$ anterior mitral leaflet excursion angle; $\beta_{E X}$, posterior mitral leaflet excursion angle; $A M L_{E X}$, anterior mitral leaflet excursion; $P M L_{E X}$, posterior mitral leaflet excursion. 
tethering angle ratio was a quantitative measurement of tethering: the more this ratio approached 1 , the more symmetric the tethering.

\section{Statistical Analysis}

Variables were tested for normal distribution by using the Kolmogorov-Smirnov test. Continuous data were expressed as means \pm standard deviation, nonparametric data were presented as medians and IQR, and frequencies were presented as proportions.

Data were compared by using the Student $t$ test, the Mann-Whitney $U$ test, the $\chi^{2}$ test, and the Fisher exact test, where appropriate. Echocardiographic variables over time were analyzed by means of repeated-measures analysis of variance, followed by the Tukey post-hoc test. Kruskal-Wallis and Friedman tests were used when variables were not normally distributed, and the Dunn post-hoc test was used for multiple comparisons.

Multivariable logistic regression analysis by means of a backward stepwise algorithm (cutoff for entry, 0.05; cutoff for removal, 0.10 ) was performed to select independent predictors of recurrent regurgitation, and 40 demographic, clinical, and echocardiographic parameters were investigated for their predictive value. To enhance the accuracy of the model, the number of variables was reduced by using variable clustering ${ }^{16}$ until the number of variables to use as candidates in the regression analysis was $\mathrm{m} / 10$ or less, where, for binary outcome, $\mathrm{m}$ is the number of patients in the less frequent outcome category. ${ }^{17}$

Model assumptions (linearity and additivity assumptions) were checked by using piecewise cubic polynomials (spiline functions) and the pooled interaction test, ${ }^{18}$ respectively, and found to be satisfied. Goodness of fit of the final logistic regression models was assessed with the Hosmer-Lemeshow statistic, ${ }^{19}$ and predictive accuracy was assessed by using the concordance index $(c) .{ }^{18}$ Internal validation of predictors generated by means of multivariable logistic regression was performed by means of bootstrapping techniques with 1000 cycles and generation of odds ratios and biascorrected $95 \%$ confidence intervals (CIs). ${ }^{18}$ Optimal cutoff values were determined as the rounding cutoff value that provides the maximum sum of sensitivity and specificity. This value should be the shoulder at the top left of the receiver operating characteristic curve. Bootstrapping techniques were used to validate the results.

SPSS 12.0 (SPSS, Chicago, Ill) and Stats Direct 2.5.7 (Stats Direct, Sale, United Kingdom) software were used for these calculations.

\section{Results}

\section{Preoperative MR and PM Displacement Leaflet Tethering}

There was no significant difference between the groups regarding qualitative and quantitative MR data (Table 2). However, in group $1 \mathrm{MR}$ jet direction was posterior in most patients, whereas it was central or anterior in most of the patients in the group 2. Furthermore, patients with recurrent MR had greater preoperative posterior and lateral displacement of the APM (both $P<.001$ ), a wider PM separation $(P=.007)$, and a larger WMSI of the segments underlying the APM $(P=.01)$.
By contrast, the WMSI of the segments underlying the PPM was higher in patients who did not have MR at postoperative control $(P=.03)$, whereas posterior and lateral displacement of the PPM were comparable in the 2 groups $(P>.9$ and $P=.7$, respectively). Moreover, tenting area $(P<.001)$ and coaptation height $(P=.004)$ were higher and $\mathrm{CL}$ was lower $(P=.006)$ in patients with recurrent MR. Contrastingly, the coaptation distance was comparable between groups $(P=.7)$. Additionally, both the anterior tethering angle $(P=.001)$ and the bending angle $(P=.01)$ were larger in group 2 , whereas the posterior tethering angle did not differ between groups $(P=.66)$. When the degree of tethering was compared further in the 2 groups, patients without postoperative MR had a more pronounced posterior leaflet tethering $(P<.001)$, with prevalent restriction of the posterior leaflet excursion $(P<.001)$. In contrast, patients with postoperative MR had a more accentuated anterior leaflet tethering $(P<.001)$, a more restricted excursion of the anterior leaflet $(P<.001)$, and a more symmetric tethering $(P<$ $.001)$ when compared with patients in group 1 .

\section{Postoperative MR and PM Displacement Leaflet Tethering}

At postoperative control (Table 3), $96.2 \%$ of patients in group 2 had posterior MR, whereas $3.8 \%$ had central or anterior MR $(P<.001)$. Annular areas reduced from the preoperative value in both groups $(P<.001)$ and remained constant over time. In patients with no recurrent MR, at discharge, indexes of posterior displacement (PPM, $P=.03$; APM, $P=$ $.001)$ and lateral displacement (PPM, $P=.02$; APM, $P=$ $.03)$ of both PMs, PM separation $(P<.001)$, annular-papillary distance $(P=.01)$, and WMSIs (PPM, $P=.02$; APM, $P=.04)$ were reduced significantly. All these parameters remained constant in this group afterward. In group 2 all indices of displacement of both PMs, as well as WMSI at the level of the PPM, showed a not significant reduction at discharge and remained stable at early control, increasing significantly at late examination. In contrast, WMSI of the left ventricular segments underlying the APM did not show any significant change over time.

Furthermore, the coaptation distance reduced significantly in both groups at discharge; however, although it kept constant in patients without recurrent MR, it significantly increased at late study in those with recurrent $\operatorname{MR}(P=$ .008). Differently, coaptation height significantly decreased at discharge $(P<.001$ and $P=.03$ in groups 1 and 2 , respectively) and kept constant afterward in both groups. CL increased in group 1 at discharge $(P<.001)$, remaining stable over time. In group $2 \mathrm{CL}$ increased at discharge $(P=.02)$ but decreased at early $(P=.01)$ and late $(P<$ $.001)$ controls. However, CL was always higher in group 1 $(P<.001)$, which included $100 \%$ of patients with CLs of $8 \mathrm{~mm}$ or greater at postoperative controls. At discharge, in both groups the anterior leaflet tethering angle significantly 
TABLE 2. Preoperative mitral regurgitation

\begin{tabular}{|c|c|c|c|c|c|}
\hline & Control subjects, $n=50$ & All, $\mathbf{n}=\mathbf{2 3 0}$ & Group1 (MR-), $n=176$ & Group $2(M R+), n=54$ & $P$ value \\
\hline \multicolumn{6}{|l|}{ MR } \\
\hline Grade & - & $3(3-4)$ & $3(3-4)$ & $3(3-4)$ & $>.9$ \\
\hline $2+$ & - & $38(16.6)$ & $29(16.4)$ & $9(16.6)$ & \\
\hline $3+$ & - & $131(56.9)$ & $99(56.3)$ & 32 (59.2) & \\
\hline $4+$ & - & $61(26.5)$ & $48(27.3)$ & $13(24.2)$ & \\
\hline \multicolumn{6}{|l|}{ Quantitative data } \\
\hline ERO & - & $37 \pm 12$ & $36 \pm 11$ & $38 \pm 14$ & .77 \\
\hline $\mathrm{RF}$ & - & $46 \pm 13$ & $44 \pm 12$ & $47 \pm 13$ & .8 \\
\hline RV & - & $57 \pm 17$ & $56 \pm 15$ & $58 \pm 18$ & .85 \\
\hline \multicolumn{6}{|l|}{ Direction of regurgitant jet } \\
\hline Central & - & $55(23.9)$ & $28(16.0)$ & $27(50.0)$ & $<.001$ \\
\hline Anterior & - & $14(6.1)$ & 7 (3.9) & $7(13.0)$ & $<.001$ \\
\hline Posterior & - & $98(42.6)$ & $89(50.6)$ & $9(16.7)$ & $<.001$ \\
\hline Complex & - & $63(27.4)$ & $52(29.5)$ & $11(20.3)$ & .12 \\
\hline \multicolumn{6}{|l|}{ Carpentier's classification ${ }^{7}$} \\
\hline Type IIIb & - & $145(64.0)$ & $110(62.3)$ & $35(64.8)$ & .8 \\
\hline Type IIIb + annular dilatation & - & $85(36.0)$ & $66(37.5)$ & $19(35.2)$ & .76 \\
\hline \multicolumn{6}{|l|}{ Mitral area } \\
\hline $\mathrm{MA}_{\mathrm{s}}$ & $4.5 \pm 0.4$ & $8.5 \pm 1.3^{*}$ & $8.1 \pm 1.2^{*}$ & $8.7 \pm 1.3^{*}$ & .41 \\
\hline $\mathrm{MA}_{\mathrm{d}}$ & $7.0 \pm 0.6$ & $11.2 \pm 1.2^{*}$ & $11.3 \pm 1.3^{*}$ & $11.7 \pm 1.1^{*}$ & $>.9$ \\
\hline $\mathrm{MA}_{\text {contr }}$ & $35 \pm 8$ & $27 \pm 3^{*}$ & $28 \pm 2^{*}$ & $27 \pm 3^{*}$ & .9 \\
\hline \multicolumn{6}{|l|}{ Papillary muscle displacement } \\
\hline PPM posterior D & $1.6 \pm 0.2$ & $2.5 \pm 0.5^{*}$ & $2.5 \pm 0.6^{*}$ & $2.5 \pm 0.3^{*}$ & $>0.9$ \\
\hline APM posterior D & $2.0 \pm 0.2$ & $2.9 \pm 0.5^{*}$ & $2.6 \pm 0.4^{*}$ & $3.2 \pm 0.5^{*}$ & $<.001$ \\
\hline PPM lateral D & $1.4 \pm 0.2$ & $1.9 \pm 0.3^{*}$ & $2.0 \pm 0.4^{*}$ & $1.8 \pm 0.3^{*}$ & .76 \\
\hline APM lateral D & $0.9 \pm 0.2$ & $1.2 \pm 0.4^{*}$ & $1.1 \pm 0.3^{*}$ & $1.5 \pm 0.4^{*}$ & $<.001$ \\
\hline PM separation & $2.5 \pm 0.4$ & $3.4 \pm 0.5^{*}$ & $3.2 \pm 0.4^{*}$ & $3.7 \pm 0.6^{*}$ & .007 \\
\hline PPM-fibrosa D & $5.1 \pm 0.5$ & $6.5 \pm 0.6^{*}$ & $6.6 \pm 0.6^{*}$ & $6.5 \pm 0.5^{*}$ & .9 \\
\hline PPM WMSI & $1.0 \pm 0$ & $2.1 \pm 0.6^{*}$ & $2.3 \pm 0.5^{*}$ & $2.0 \pm 0.5^{*}$ & .03 \\
\hline APM WMSI & $1.0 \pm 0$ & $14 \pm 0.3^{*}$ & $1.3 \pm 0.1^{*}$ & $1.6 \pm 0.4^{*}$ & .01 \\
\hline \multicolumn{6}{|l|}{ Coaptation } \\
\hline TA & $0.8 \pm 2$ & $3.5 \pm 1.1^{*}$ & $3.1 \pm 1.0^{*}$ & $4.1 \pm 1.1^{*}$ & $<.001$ \\
\hline$C L$ & $8.5 \pm 0.4$ & $3.5 \pm 0.4^{*}$ & $4.0 \pm 0.5^{*}$ & $3.3 \pm 0.3^{*}$ & .006 \\
\hline$d$ & $27.6 \pm 6$ & $35.1 \pm 7^{*}$ & $34.8 \pm 9^{*}$ & $36.4 \pm 6^{*}$ & .7 \\
\hline $\mathrm{h}$ & $6.1 \pm 2$ & $11.3 \pm 3^{*}$ & $9.5 \pm 3^{*}$ & $13.1 \pm 4^{*}$ & .004 \\
\hline \multicolumn{6}{|l|}{ Mitral leaflet tethering } \\
\hline$\alpha^{\prime}$ & $24.3 \pm 3$ & $39.0 \pm 5^{*}$ & $34.3 \pm 5^{*}$ & $44.0 \pm 9$ & .001 \\
\hline$\gamma$ & $156.6 \pm 12$ & $138 \pm 10^{*}$ & $144 \pm 12^{*}$ & $130 \pm 9^{*}$ & .01 \\
\hline$\beta^{\prime}$ & $33.4 \pm 5$ & $52.0 \pm 12^{*}$ & $54.0 \pm 11^{*}$ & $50.0 \pm 14^{*}$ & .66 \\
\hline$\alpha^{\prime} / \beta^{\prime}$ ratio & $0.72 \pm 0.4$ & $0.75 \pm 0.1$ & $0.63 \pm 0.1^{*}$ & $0.88 \pm 0.1^{*}$ & $<.001$ \\
\hline \multicolumn{6}{|l|}{ Leaflet excursion } \\
\hline$\alpha_{\mathrm{EX}}$ & $46.0 \pm 7$ & $31 \pm 4^{*}$ & $36.0 \pm 5^{*}$ & $26.1 \pm 4$ & .01 \\
\hline$\beta_{\mathrm{EX}}$ & $28.6 \pm 4$ & $10 \pm 5^{*}$ & $10 \pm 2^{*}$ & $12 \pm 4^{*}$ & .52 \\
\hline$A M L_{E X}$ & $21.5 \pm 5$ & $14.4 \pm 3^{*}$ & $17.6 \pm 3^{*}$ & $13.1 \pm 3^{*}$ & .003 \\
\hline$P M L_{E X}$ & $9.5 \pm 0.7$ & $3.6 \pm 0.3$ & $3.5 \pm 0.2^{*}$ & $4.0 \pm 0.2^{*}$ & .2 \\
\hline
\end{tabular}

Continuous variables are presented as means \pm standard deviation, and nonparametric variables are presented as medians (interquartile range). $P$ values indicate significance between groups. ${ }^{*}$ Significance versus control subjects. $M R$, Mitral regurgitation; $E R O$, effective regurgitant orifice $\left(\mathrm{mm}^{2}\right) ; R F$, regurgitant fraction (\%); $R V$, regurgitant volume (mL/beat); $M A_{s}$, systolic mitral annular area $\left(\mathrm{cm}^{2} / \mathrm{m}^{2}\right) ; M A_{d}$ diastolic mitral annular area $\left(\mathrm{cm}^{2} / \mathrm{m}^{2}\right) ; M A_{c o n t n}$ mitral annular contraction (\%); $P P M$, posterior papillary muscle; $D$, distance $(\mathrm{cm}) ; A P M$, anterior papillary muscle; $P M$ separation, separation of papillary muscles $(\mathrm{cm})$; WMSI, wall motion score index; TA, tenting area $\left(\mathrm{cm}^{2}\right) ; C L$, coaptation length $(\mathrm{mm}) ; d$, coaptation distance $(\mathrm{mm}) ; h$, coaptation height $(\mathrm{mm}) ; \alpha^{\prime}$, anterior mitral leaflet tethering angle $\left({ }^{\circ}\right) ; \gamma$, bending angle $\left({ }^{\circ}\right) ; \beta^{\prime}$, posterior mitral leaflet tethering angle $\left({ }^{\circ}\right) ; \alpha_{E X}$, anterior mitral leaflet excursion angle $\left({ }^{\circ}\right) ; \beta_{E X}$, posterior mitral leaflet excursion angle ( ${ }^{\circ}$ ); $A M L_{E X}$, anterior mitral leaflet excursion (mm); $P M L_{E X}$, posterior mitral leaflet excursion (mm). 
TABLE 3. Postoperative mitral regurgitation

\begin{tabular}{|c|c|c|c|c|c|c|c|c|c|}
\hline & \multirow{2}{*}{$\begin{array}{c}\text { Control subjects, } \\
\quad n=50\end{array}$} & \multicolumn{4}{|c|}{ Group1 (MR-), n = 176} & \multicolumn{4}{|c|}{ Group $2(\mathrm{MR}+), \mathrm{n}=54$} \\
\hline & & Discharge & Early & Late & $P$ value & Discharge & Early & Late & $P$ value \\
\hline MR grade & - & $0(0-1)$ & $0(0-1)$ & $0(0-1)$ & $>.9$ & $0(0-1)$ & $2(2-3) \dagger 9$ & $3(3-4) \ddagger^{\#}$ & $<.001$ \\
\hline None/trace & - & $176(100)$ & $176(100)$ & $176(100)$ & & $54(100)$ & $5(9.2)$ & - & \\
\hline $2+$ & - & - & - & - & & - & $24(44.5)$ & $12(22.3)$ & \\
\hline $3+$ & - & - & - & - & & - & $24(44.5)$ & $34(62.9)$ & \\
\hline $4+$ & - & - & - & - & & - & $1(1.8)$ & $8(14.8)$ & \\
\hline \multicolumn{10}{|l|}{ Quantitative data } \\
\hline ERO & - & - & - & - & - & - & $16 \pm 7$ & $32 \pm 11$ & $<.001$ \\
\hline $\mathrm{RF}$ & - & - & - & - & & - & $24 \pm 5$ & $44 \pm 13$ & $<.001$ \\
\hline RV & - & - & - & - & & - & $28 \pm 5$ & $56 \pm 18$ & $<.001$ \\
\hline \multicolumn{10}{|c|}{$\begin{array}{l}\text { Direction of regurgitant } \\
\text { jet }\end{array}$} \\
\hline Central & - & - & - & - & & - & - & - & \\
\hline Anterior & - & - & - & - & & - & - & - & \\
\hline Posterior & - & - & - & - & & - & $52(96.2)$ & $52(96.2)$ & \\
\hline Complex & - & - & - & - & & - & $2(3.8)$ & $2(3.8)$ & \\
\hline \multicolumn{10}{|l|}{ Mitral area } \\
\hline $\mathrm{MA}_{\mathrm{s}}$ & $4.5 \pm 0.4$ & $4.9 \pm 1.3^{*}$ & $4.9 \pm 1.2$ & $4.9 \pm 1.3$ & .6 & $5.0 \pm 1.3^{*}$ & $5.1 \pm 1.2$ & $5.1 \pm 1.3$ & .7 \\
\hline$M A_{d}$ & $7.0 \pm 0.6$ & $6.4 \pm 2.2^{*}$ & $6.4 \pm 2.3$ & $6.4 \pm 2.3$ & .5 & $6.3 \pm 2.2^{*}$ & $6.3 \pm 2.3$ & $6.3 \pm 2.0$ & $>.9$ \\
\hline $\mathrm{MA}_{\text {contr }}$ & $35 \pm 8$ & $23 \pm 3^{*}$ & $23 \pm 2$ & $23 \pm 3$ & .34 & $20 \pm 3^{*}$ & $19 \pm 2$ & $19 \pm 3$ & .79 \\
\hline \multicolumn{10}{|l|}{$\begin{array}{l}\text { Papillary muscle } \\
\text { displacement }\end{array}$} \\
\hline PPM posterior D & $1.6 \pm 0.2$ & $2.2 \pm 0.3^{*}$ & $2.2 \pm 0.3$ & $2.2 \pm 0.2$ & $>.9$ & $2.5 \pm 0.3 \S$ & $2.4 \pm 0.39$ & $2.7 \pm 0.4 t_{\ddagger}^{\#}$ & $<.001$ \\
\hline APM posterior D & $2.0 \pm 0.2$ & $2.3 \pm 0.4^{*}$ & $2.3 \pm 0.4$ & $2.3 \pm 0.4$ & $>.9$ & $3.1 \pm 0.5 \S$ & $3.0 \pm 0.59$ & $3.4 \pm 0.6 t_{\dagger}^{\#}$ & .005 \\
\hline PPM lateral D & $1.4 \pm 0.2$ & $1.8 . \pm 0.3^{*}$ & $1.7 \pm 0.3$ & $1.7 \pm 0.3$ & .88 & $1.8 \pm 0.3$ & $1.8 \pm 0.39$ & $2.4 \pm 0.3 \ddagger^{\#}$ & .002 \\
\hline APM lateral D & $0.9 \pm 0.2$ & $0.9 \pm 0.2^{*}$ & $0.9 \pm 0.3$ & $0.9 \pm 0.3$ & .86 & $1.4 \pm 0.4 \S$ & $1.4 \pm 0.59$ & $1.6 \pm 0.4 \ddagger^{\#}$ & .02 \\
\hline PM separation & $2.5 \pm 0.4$ & $3.0 \pm 0.4^{*}$ & $2.9 \pm 0.2$ & $2.9 \pm 0.2$ & .9 & $3.6 \pm 0.6 \S$ & $3.6 \pm 0.69$ & $3.8 \pm 0.7 \ddagger^{\#}$ & .04 \\
\hline PPM-fibrosa D & $5.1 \pm 0.5$ & $6.1 \pm 0.5^{*}$ & $6.2 \pm 0.6$ & $6.1 \pm 0.5$ & .88 & $6.5 \pm 0.5$ & $6.4 \pm 0.6$ & $7.1 \pm 0.7 \ddagger_{\ddagger}^{\#}$ & $<.001$ \\
\hline PPM WMSI & $1.0 \pm 0$ & $2.1 \pm 0.3^{*}$ & $2.2 \pm 0.4$ & $2.3 \pm 0.3$ & .69 & $1.9 \pm 0.5 \S$ & $1.9 \pm 0.6$ & $2.4 \pm 0.4 \ddagger$ & .02 \\
\hline APM WMSI & $1.0 \pm 0$ & $1.2 \pm 01^{*}$ & $1.2 \pm 0.1$ & $1.2 \pm 0.1$ & $>.9$ & $1.5 \pm 0.4 \S$ & $1.5 \pm 0.36$ & $1.5 \pm 0.4^{\#}$ & $>.9$ \\
\hline \multicolumn{10}{|l|}{ Coaptation } \\
\hline TA & $0.8 \pm 2$ & $2.6 \pm 1.1^{*}$ & $2.7 \pm 1.0$ & $2.7 \pm 1.1$ & .9 & $3.5 \pm 1.1 * \S$ & $3.6 \pm 1.09$ & $3.6 \pm 1.1^{\#}$ & $>.9$ \\
\hline $\mathrm{CL}$ & $8.5 \pm 0.4$ & $8.4 \pm 0.4^{*}$ & $8.5 \pm 0.5$ & $8.5 \pm 0.3$ & .8 & $5.1 \pm 0.4^{*} \S$ & $4.1 \pm 0.3 \dagger 9$ & $3.0 \pm 0.3 \ddagger^{\#}$ & .9 \\
\hline $\mathrm{d}$ & $27.6 \pm 6$ & $31.1 \pm 7^{*}$ & $32.8 \pm 9$ & $33.4 \pm 6$ & .08 & $33.1 \pm 7^{*} \S$ & $35.0 \pm 99$ & $38.4 \pm 6 \ddagger^{\#}$ & .01 \\
\hline $\mathrm{h}$ & $6.1 \pm 2$ & $7.3 \pm 3^{*}$ & $7.5 \pm 3$ & $7.1 \pm 3$ & .06 & $11 \pm 3^{*} \S$ & $11.5 \pm 39$ & $11.1 \pm 4^{\#}$ & $>.9$ \\
\hline \multicolumn{10}{|c|}{ Mitral leaflet tethering } \\
\hline$\alpha^{\prime}$ & $24.3 \pm 3$ & $25.5 \pm 7^{*}$ & $23.2 \pm 5$ & $24.0 \pm 6$ & 6 & $37.4 \pm 8^{*} \S$ & $35.8 \pm 89$ & $35.2 .0 \pm 9^{\#}$ & .79 \\
\hline$\gamma$ & $156.6 \pm 12$ & $151.2 \pm 10^{*}$ & $150.3 \pm 8$ & $150.6 \pm 9$ & .9 & $124.1 \pm 6^{*} \S$ & $124.3 \pm 69$ & $120.1 \pm 5^{\#}$ & .8 \\
\hline$\beta^{\prime}$ & $33.4 \pm 5$ & $62.0 \pm 12^{*}$ & $64.3 \pm 11$ & $79.1 \pm 14 \ddagger$ & .001 & $62.3 \pm 12^{*}$ & $66.2 \pm 11 \dagger 9$ & $76.5 \pm 14 \ddagger$ & $<.001$ \\
\hline$\alpha^{\prime} / \beta^{\prime}$ ratio & $0.72 \pm 0.4$ & $0.41 \pm 0.1^{*}$ & $0.36 \pm 0.1$ & $0.30 \pm 0.1$ & $<.001$ & $0.60 \pm 0.2^{*}$ & $0.54 \pm 0.2 \uparrow \Phi$ & $0.46 \pm 0.1 \dagger^{\#}$ & $<.001$ \\
\hline \multicolumn{10}{|l|}{ Leaflet excursion } \\
\hline$\alpha_{\mathrm{EX}}$ & $46.0 \pm 7$ & $40.3 \pm 4^{*}$ & $41.0 \pm 4$ & $42.6 \pm 5$ & .7 & $34.0 \pm 3^{*} \S$ & $34.0 \pm 39$ & $34.1 \pm 4^{\#}$ & $>.9$ \\
\hline$\beta_{\mathrm{EX}}$ & $28.6 \pm 4$ & $6 \pm 2^{*}$ & $6 \pm 2$ & $4 \pm 4 \ddagger$ & .03 & $6 \pm 3^{*}$ & $6 \pm 2$ & $4 \pm 4 \ddagger$ & .03 \\
\hline$A M L_{E X}$ & $21.5 \pm 5$ & $19.4 \pm 3^{*}$ & $19.6 \pm 3$ & $20.3 \pm 4$ & .64 & $16.6 \pm 3^{*} \S$ & $16.1 \pm 39$ & $16.2 \pm 3^{\#}$ & .9 \\
\hline$P M L_{E X}$ & $9.5 \pm 0.7$ & $2.7 \pm 0.3$ & $2.7 \pm 0.2$ & $1.4 \pm 0.2 \ddagger$ & .001 & $3.4 \pm 0.3^{*}$ & $3.1 \pm 0.2$ & $2.0 \pm 0.2 \ddagger$ & $<.001$ \\
\hline
\end{tabular}

Continuous variables are presented as means \pm standard deviation, and nonparametric variables are presented as medians (interquartile range). $P$ values indicate significance over time. $M R$, Mitral regurgitation; $E R O$, effective regurgitant orifice $\left(\mathrm{mm}^{2}\right) ; R$, regurgitant fraction $(\%) ; R V$, regurgitant volume $(\mathrm{mL} /$ beat); $M A_{s}$, systolic mitral annular area $\left(\mathrm{cm}^{2}\right) ; M A_{d}$, Diastolic mitral annular area $\left(\mathrm{cm}^{2}\right) ; M A_{\text {contn }}$ mitral annular contraction $(\%) ; P P M$, posterior papillary muscle; $D$, distance $(\mathrm{cm}) ; A P M$, anterior papillary muscle; $P M$ separation, separation of papillary muscles $(\mathrm{cm}) ; W M S I$, wall motion score index; $T A$, tenting area $\left(\mathrm{cm}^{2}\right) ; C L$, coaptation length $(\mathrm{mm}) ; d$, coaptation distance $(\mathrm{mm}) ; h$, coaptation height $(\mathrm{mm}) ; \alpha^{\prime}$, anterior mitral leaflet tethering angle $\left({ }^{\circ}\right) ; \gamma$, bending angle $\left({ }^{\circ}\right) ; \beta^{\prime}$, posterior mitral leaflet tethering angle $\left({ }^{\circ}\right) ; \alpha_{E X}$, anterior mitral leaflet excursion angle $\left(^{\circ}\right) ; \beta_{E X}$, posterior mitral leaflet excursion angle $\left({ }^{\circ}\right) ; A M L_{E X}$, anterior mitral leaflet excursion (mm); $P M L_{E X}$, posterior mitral leaflet excursion (mm). $\S \rrbracket \#$ Significance vs group 1 at discharge, early, and late control, respectively. ${ }^{*} \dagger \ddagger S i g n i f i c a n c e$ versus preoperative, discharge and early follow-up, respectively. 
decreased $(P=.01$ and $P=.03$ in groups 1 and 2, respectively), whereas the excursion angle $(P=.02$ and $P<$ .001 in groups 1 and 2, respectively) and the excursion distance of the anterior leaflet increased (both $P=.02$ ). All these indices remained constant afterward. In contrast, the posterior leaflet tethering angle increased at discharge (both $P<.001$ ) whereas the excursion angle (both $P<.001$ ) and excursion distance (both $P<.001$ ) decreased significantly in both groups. These indices remained stable at early control, but at late echocardiography, the tethering angle increased again (both $P<.001$ ), whereas the excursion angle (both $P=.03$ ) and the excursion distance (both $P<.001$ ) decreased without a difference between the groups.

The bending angle significantly increased at discharge in patients without postoperative MR $(P=.03)$ and kept constant at subsequent controls. Differently, the bending angle showed a significant decrease in patients with postoperative MR $(P=.008)$, remaining constant afterward. When the degree of tethering was examined further in the 2 groups, it became progressively more asymmetric in both groups (group 1: $P<.001$ vs preoperative, $P=.03$ vs discharge, and $P=.03$ vs early; group $2: P<.001$ vs preoperative, $P=.03$ vs discharge, and $P=.02$ vs early).

\section{Valvular and Subvalvular Determinants of Recurrent MR}

Multivariable regression analysis (Table 4) identified an anterior tethering angle of $39.5^{\circ}$ or greater $(P<.001)$, an anterior/posterior tethering angle ratio of 0.76 or greater $(P<$ $.001)$, an anterior leaflet excursion angle of $35^{\circ}$ or less $(P=.001)$, and a coaptation height $(h)$ of $11 \mathrm{~mm}$ or greater $(P=.04)$ as independent predictors of recurrent MR. Internal validation of such multivariable analysis by means of bootstrapping, including the same variables of standard logistic regression model, further confirmed the significant predictive nature of early death (bootstrap odds ratio, 1.7; bias-corrected 95\% CI, 0.6-3.8). Finally, the logistic regression model was reliable (Hosmer-Lemeshow test, $p=.6$ ) and accurate $(c$ index $=0.8)$.

\section{Discussion}

The main purpose of the present study was to investigate leaflet configurations in patients with CIMR before and after combined reductive annuloplasty and CABG and to identify characteristics of valvular, as well as subvalvular, configurations responsible for recurrent ischemic regurgitation. Clinical data, as well further treatment options offered for patients with discovery of residual/recurrent MR, have been previously reported ${ }^{9}$ and have not been included in the present study.

\section{Subvalvular Mechanism of Recurrent MR}

Previous studies ${ }^{7,20}$ demonstrated that the local remodeling of the left ventricular segments supporting the PPM is a necessary condition for the development of MR. Accordingly, we found that remodeling of the regions supporting the PPM occurred in both groups, as demonstrated by the WMSIs of these regions, which were higher than normal in all patients. Nonetheless, in our experience patients with recurrent MR had preoperatively a greater local left ventricular remodeling of the left ventricular segments underlying the APM, a greater lateral and posterior displacement of the APM, and a wider PM separation.

Interestingly, this different distribution of local wall abnormalities did not reflect, in our experience, a different infarct localization, confirming that mechanisms underlying CIMR are complex and not completely understood. However, our results support previous clinical findings reported by Agricola and colleagues, ${ }^{15}$ who described 2 groups of patients with CIMR. Like group 2 in our study, their symmetric group had prevalent posterior and lateral displacement of APM (both $P<.04$ ), PM separation, and WMSI of the regions supporting the APM (both $P<.001$ ).

After surgical intervention, we showed that continued left ventricular local remodeling occurs in both groups, and it predominantly involves the region of the left ventricle supporting the PPM, whereas the WMSI of segments underlying the APM remained constant over time postoperatively in the entire cohort.

\section{Valvular Mechanism of Recurrent MR}

This study has demonstrated different baseline leaflet configurations in patients with and without recurrent MR. Patients without postoperative MR had a more asymmetric tethering pattern with a more pronounced PML tethering and with a prevalent restriction of the posterior leaflet excursion.

TABLE 4. Multivariable analysis

\begin{tabular}{|c|c|c|c|c|c|c|c|c|c|}
\hline Variable & Cutoff & $95 \% \mathrm{Cl}^{*}$ & Sensitivity & Specificity & AUC & $95 \% \mathrm{CI}$ & OR & Bias-corrected $95 \% \mathrm{Cl}$ & $P$ value \\
\hline$\alpha^{\prime}$ & $\geq 39.5^{\circ}$ & $34.3^{\circ}-41.2^{\circ}$ & $98 \%$ & $97 \%$ & 0.99 & $0.92-1$ & 5.4 & $2.6-6.9$ & $<.001$ \\
\hline$\alpha^{\prime} / \beta^{\prime}$ & $\geq 0.76$ & $0.68-0.81$ & $87 \%$ & $86 \%$ & 0.92 & $0.89-0.95$ & 4.8 & $2.0-6.3$ & $<.001$ \\
\hline$\alpha_{\mathrm{EX}}$ & $\leq 35^{\circ}$ & $32^{\circ}-39^{\circ}$ & $85 \%$ & $83 \%$ & 0.87 & $0.81-0.93$ & 2.3 & $1.2-3.1$ & .001 \\
\hline $\mathrm{h}$ & $\geq 11 \mathrm{~mm}$ & $8.7-11.9$ & $81 \%$ & $84 \%$ & 0.89 & $0.83-0.95$ & 1.8 & $0.8-2.3$ & .04 \\
\hline
\end{tabular}

$\mathrm{Cl}$, Confidence interval; $A U C$, area under curve; $\alpha^{\prime}$, anterior mitral leaflet tethering angle; $\beta^{\prime}$, posterior mitral leaflet tethering angle $\left({ }^{\circ}\right) ; \alpha_{E X}$, anterior mitral leaflet excursion angle; $h$, coaptation height. ${ }^{*} 95 \% \mathrm{Cl}$ determined by means of bootstrapping. 
Contrastingly, patients with postoperative MR had a more symmetric tethering pattern, a more accentuated anterior leaflet tethering, a more restricted anterior leaflet excursion, and a smaller bending angle. The different tethering pattern translated into different preoperative MR jet directions: predominantly posterior in group 1 and mainly central in group 2 . Postoperatively, the tethering of the posterior leaflet increased, and it was predominant in both groups at any stage postoperatively. In contrast, the tethering of the anterior leaflet reduced and the AML excursion angle increased significantly early after surgical intervention, remaining constant afterward in both groups. This is consistent with the report by Zhu and associates, ${ }^{20}$ who observed a prevalent posterior leaflet tethering either in patients with $(\mathrm{n}=25)$ or without $(\mathrm{n}=6)$ postoperative MR $(P<.05)$. Tethering of the posterior leaflet can be exacerbated by annuloplasty, which, by reducing annular size, can potentially shift the posterior annulus farther anteriorly, increasing its displacement relative to the PMs without displacing the anterior annulus fixed at the aortic root. ${ }^{21}$ However, patients in group 2 showed a mechanism of recurrent MR different from that of MR at baseline, with a tethering mechanism becoming progressively more asymmetric. In contrast with Kuwahara and associates ${ }^{8}$ and Magne and coworkers, ${ }^{22}$ in our experience tethering of the posterior leaflet was not significant as a predictor of MR recurrence. However, these authors studied recurrent/persistent and persistent MR, respectively, whereas in our study residual MR was excluded. Persistent/residual MR is presumably caused by immediate failure of the technique used rather than left ventricular remodeling, ${ }^{23}$ and it is not surprising that regurgitation persists at late follow-up in these patients. Contrastingly, we found an anterior tethering angle of $39.5^{\circ}$ or greater $(P<.001)$, an anterior/posterior tethering angle ratio of 0.76 or greater $(P<.001)$, an anterior leaflet excursion angle of $35^{\circ}$ or less $(P=.001)$, and a coaptation height $(h)$ of $11 \mathrm{~mm}$ or greater $(P=.04)$ to be independent predictors of recurrent MR. Therefore in accordance with Calafiore and colleagues, ${ }^{24}$ a coaptation distance (in our study coaptation height) of $11 \mathrm{~mm}$ or greater was associated with a high risk of MR recurrence after UMRA. Nevertheless, as far as we know, this is the first demonstration that patients with predominant tethering of the AML had a higher likelihood of postoperative recurrent MR after reductive annuloplasty. We can postulate that patients with more accentuated AML tethering cannot guarantee a sufficiently long anterior leaflet to cover the whole anteroposterior diameter, and this is worsened by the augmented postoperative tethering of the posterior leaflet, which further displaces the coaptation point posteriorly. Green and associates ${ }^{21}$ have reported, in an ovine model, that annuloplasty produces a markedly impaired motion of a "frozen" PML, transforming the mitral valve into a unicuspid valve, where the mechanism of closure is performed only by the anterior leaflet, and Kuwahara and associates $^{8}$ concluded that an anterior leaflet longer than the anteroposterior annular diameter is required in the presence of a restricted posterior leaflet to ensure successful repair. However, in our opinion a long anterior leaflet could not always be sufficient to balance tethering forces caused by postoperative remodeling, and the $\mathrm{CL}$ gained at the end of procedure could play a significant role in preventing MR recurrence. Indeed, patients in group 1 had larger CLs $(P<$ .001 ), and $100 \%$ showed a CL of $8 \mathrm{~mm}$ or greater at postoperative controls. Interestingly, a subgroup of patients in group 2 showed postoperative MR, even with a less tethered AML: in one of them the CL was small $(2.6 \mathrm{~mm})$, whereas in 2 the apposition surface was absent and the AML closed against the annulus. Thus on the basis of actual experience, we believe that in all subjects, especially those who are expected not to reverse remodel, a minimum CL of $8 \mathrm{~mm}$ should be achieved, as suggested by other authors. ${ }^{25}$ Nonetheless, in patients with strongly tethered AMLs or with less tethered anterior leaflets not sufficiently long to ensure a postoperative CL of $8 \mathrm{~mm}$ or greater, MR presumably cannot be eliminated by means of ring annuloplasty. In these patients a technique directly addressing left ventricular remodeling or a chordal sparing mitral valve replacement should be considered. Additionally, a key point for achieving an appropriate leaflet coaptation and to ensure durable results is a true undersizing, and in this setting a pivotal role can be played by the type of ring chosen. In the present experience we used only 2 rings (Carpentier's rigid or Physio semiflexible rings; Edwards LifeSciences, Irvine, Calif). These 2 rings are not at all identical, and thus even though we have undersized both rings by 2 sizes, we might have been less restrictive with the Physio ring than with the Classic ring. However, in our previous experience $^{9}$ neither ring type nor ring size were predictive of recurrent MR.

Finally, patients with a more accentuated AML tethering and restrictive excursion could benefit from anterior basal chord cutting as a method of improving leaflet mobility and decreasing leaflet tethering. Nonetheless, we have no experience with this technique, and this issue deserves further investigation.

\section{Limitations of the Study}

Our study should be viewed in light of some inherent limitations. First, evaluation of mechanisms of recurrent ischemic MR was based on geometric assumptions and 2-dimensional echocardiographic measurements that are subject to imageplane positioning errors. Hence it might be not accurate in shape-distorted postinfarction left ventricles. The era of 3dimensional echocardiography has started potentially leading to new insights into left ventricular remodeling, mitral apparatus shape, and function. ${ }^{26}$ Nonetheless, the 3 -dimensional method is still on its way and is far from being validated, standardized, and widely applied in clinical practice. ${ }^{27}$

Second, viability testing was not performed in these patients. Therefore recurrence of MR might be also due to 
irreversible ischemic myocardial damage (nonviable myocardium). Furthermore, in relation to the PM displacement data presented, the revascularization of viable regions related to PM function might be the most important predictor of success with this operation. This limitation is shared with most published article. However, this issue deserves further investigation.

Third, postoperative evaluation of coronary status was not assessed. It would have been helpful to differentiate between surgical failure (valve repair and CABG) and the progress of the coronary disease.

Fourth, the issue of annulus reshaping during annuloplasty has not been addressed. We used only rigid/semiflexible plane annuloplasty rings, which can flatten the natural saddle shape, causing greater tension on chordae and leaflets. Innovative annular rings have been recently introduced to the market that mimic the shape of healthy mitral annulus. ${ }^{1,28-30}$

Fifth, estimated cutoff values are known to be very susceptible to changes in the study population. We used bootstrapping techniques to validate the results; nonetheless, it has also been documented that the sensitivities/specificities associated with these cutoffs are overly optimistic.

\section{Strength of the Study}

The study does not display most of the common clinical and methodological pitfalls that limit results from many studies of mitral valve repair. ${ }^{23}$ The patient cohort was large, and the echocardiographic follow-up was detailed and 100\% complete. In addition, our study population was more homogeneous than in other reports: all patients underwent associated $\mathrm{CABG}$, they had no concomitant mitral valve procedures, and the entire cohort was uniform regarding the mitral valve ischemic leaflet dysfunction. Moreover, only true "recurrent" MR was studied, excluding those patients with "residual" MR in whom the insufficiency was presumably never eliminated at surgical intervention. Additionally, we undertook valve sizing in a standardized fashion, and the degree of undersizing was homogeneous over the 5-year period of the study. Finally, we used only 2 rings (rigid and semiflexible), which were equally distributed in the 2 groups.

\section{Conclusions}

Preoperative symmetric tethering with AML predominance was strongly associated with recurrence of MR. Measures of leaflet tethering, which are not systematically used in clinical practice, resulted, in our experience, in fundamental findings to identify ischemic patients who can really benefit from restrictive annuloplasty. Further larger studies are necessary to confirm our findings.

We thank Dr Orlando Parise for statistical analysis. We thank Dr Judith Wilson for the English revision of the article.

\section{References}

1. Gorman JH 3rd, Jackson BM, Enomoto Y, Gorman RC. The effect of regional ischemia on mitral valve annular saddle shape. Ann Thorac Surg. 2004;77:544-8.

2. Otsuji Y, Handschumacher MD, Schwammenthal E, et al. Insights from three-dimensional echocardiography into the mechanism of functional mitral regurgitation: direct in vivo demonstration of altered leaflet tethering geometry. Circulation. 1997;96:1999-2008.

3. Bolling SF, Pagani FD, Deeb GM, Bach DS. Intermediate term outcome of mitral reconstruction in cardiomyopathy. J Thorac Cardiovasc Surg. 1998;115:381-6.

4. Bolling SF, Deeb GM, Bach DS. Mitral valve reconstruction in elderly, ischemic patients. Chest. 1996;109:35-40.

5. McGee EC, Gillinov AM, Blackstone EH, et al. Recurrent mitral regurgitation after annuloplasty for functional ischemic mitral regurgitation. J Thorac Cardiovasc Surg. 2004;128:916-24.

6. Serri K, Bouchard D, Demers P, et al. Is a good perioperative echocardiographic result predictive of durability in ischemic mitral valve repair? J Thorac Cardiovasc Surg. 2006;131:523-9.

7. Hung J, Papakostas L, Tahta SA, et al. Mechanism of recurrent ischemic mitral regurgitation after annuloplasty: continued LV remodeling as a moving target. Circulation. 2004;110(suppl II):II85-90.

8. Kuwahara E, Otsuji Y, Iguro Y, et al. Mechanism of recurrent/persistent ischemic/functional mitral regurgitation in the chronic phase after surgical annuloplasty: importance of augmented posterior leaflet tethering. Circulation. 2006;114(suppl):I529-34.

9. Gelsomino S, Lorusso R, De Cicco G, et al. Five-year echocardiographic results of combined undersized mitral ring annuloplasty and coronary artery bypass grafting for chronic ischaemic mitral regurgitation. Eur Heart J. 2008;29:231-40.

10. World Medical Association Declaration of Helsinki: ethical principles for medical research involving human subjects. Available at: http:// www.wma.net/e/policy/b3.htm. Accessed January 9, 2008.

11. Bland MJ, Altman DJ. Statistical method for assessing agreement between two methods of clinical measurements. Lancet. 1986;1:307-10.

12. Zoghbi WA, Enriquez-Sarano M, Foster E, et al. Recommendations for evaluation of the severity of native valvular regurgitation with two-dimensional and Doppler echocardiography. J Am Soc Echocardiogr. 2003; 16:777-802.

13. Grigioni F, Enriquez-Sarano M, Zehr KJ, Bailey KL, Tajik AJ. Ischemic mitral regurgitation. Long-term outcome and prognostic implications with quantitative Doppler assessment. Circulation. 2001;103:1759-64.

14. Vijayaraghavan G, Boltwood CM, Tei C, Wong M, Shah PM. Simplified echocardiographic measurement of the mitral annulus. Am Heart J. 1986;112:985-91.

15. Agricola E, Oppizzi M, Maisano F, et al. Echocardiographic classification of chronic mitral regurgitation caused by restricted motion according to tethering pattern. Eur J Echocardiogr. 2004;5:326-34.

16. Romesburg HC. Cluster analysis for researchers. Morrisville, NC: Lulu Press; 2004. p. 1-330.

17. Harrel FE, Lee KL, Califf RM, Pryor DB, Rosati RA. Regression modelling strategies for improved prognostic prediction. Stat Med. 1984;3: 143-52.

18. Harrel FE Jr, Lee KL, Mark DB. Multivariable prognostic models: issues in developing models, evaluating assumptions and adequacy, and measuring and reducing errors. Stat Med. 1996;15(4):361-87.

19. Hosmer DW, Lemeshow S. Applied logistic regression. 2nd ed. New York: Wiley-Interscience; 2000. p. 143-202.

20. Zhu F, Otsuji Y, Yotsumoto G, et al. Mechanism of persistent ischemic mitral regurgitation after annuloplasty: importance of augmented posterior mitral leaflet tethering. Circulation. 2005;112(suppl):1396-401.

21. Green GR, Dagum P, Glasson JR, et al. Restricted posterior leaflet motion after mitral ring annuloplasty. Ann Thorac Surg. 1999;68:2100-6.

22. Magne J, Pibarot P, Dagenais F, Hachicha Z, Dumesnil JG, Senechal M. Preoperative posterior leaflet angle accurately predicts outcome after restrictive mitral valve annuloplasty for ischemic mitral regurgitation. Circulation. 2007;115:782-91.

23. Adams DH, Anyanwu AC. Pitfalls and limitations in measuring and interpreting the outcomes of mitral valve repair. $J$ Thorac Cardiovasc Surg. 2006;131:523-9. 
24. Calafiore AM, Di Mauro M, Gallina S, et al. Mitral valve surgery for chronic ischemic mitral regurgitation. Ann Thorac Surg. 2004;77: 1989-97.

25. Braun J, Bax JJ, Versteegh MI, et al. Preoperative left ventricular dimensions predict reverse remodeling following restrictive mitral annuloplasty in ischemic mitral regurgitation. Eur J Cardiothorac Surg. 2005;27:847-53.

26. Tsukiji M, Watanabe N, Yamaura Y, et al. Three-dimensional quantitation of mitral valve coaptation by a novel software system with transthoracic real-time three-dimensional echocardiography. $J$ Am Soc Echocardiogr. 2008;21:43-6.
27. Ryan LP, Jackson BM, Parish LM, et al. Mitral valve tenting index for assessment of subvalvular remodeling. Ann Thorac Surg. 2007;84:1243-9.

28. Votta E, Maisano F, Bolling SF, Alfieri O, Montevecchi FM, Redaelli A. The Geoform disease-specific annuloplasty system: a finite element study. Ann Thorac Surg. 2007;84:92-101.

29. Salgo IS, Gorman JH, Gorman RC, et al. Effect of Annular Shape on Leaflet Curvature in Reducing Mitral Leaflet Stress. Circulation. 2002;106:711-7.

30. Jimenez JH, Liou SW, Padala M, et al. A saddle-shaped annulus reduces systolic strain on the central region of the mitral valve anterior leaflet. J Thorac Cardiovasc Surg. 2007;134:1562-8.

\section{Appendix 1. Bland-Altman limits of agreement for intraobserver and interobserver variability}

\begin{tabular}{|c|c|c|c|}
\hline Variable & Mean difference & Standard deviation & $95 \%$ limits of agreement \\
\hline \multicolumn{4}{|l|}{$\mathrm{ESV}(\mathrm{mL})$} \\
\hline Intraobserver (IC) & 1.3 & 1.6 & -1.8 to 4.4 \\
\hline Intraobserver (CR) & 0.5 & 2.7 & -4.8 to 5.8 \\
\hline Interobserver & 1.6 & 2.9 & -4.0 to 7.3 \\
\hline \multicolumn{4}{|l|}{$\operatorname{LVEF}(\%)$} \\
\hline Intraobserver (IC) & 2.4 & 1.8 & -1.1 to 6.0 \\
\hline Intraobserver (CR) & 2.5 & 2.0 & -1.5 to 6.5 \\
\hline Interobserver & 2.3 & 2.8 & -3.0 to 7.8 \\
\hline \multicolumn{4}{|l|}{$\mathrm{ERO}\left(\mathrm{mm}^{2}\right)$} \\
\hline Intraobserver (IC) & 0.4 & 2.1 & -3.8 to 4.7 \\
\hline Intraobserver (CR) & 0.5 & 1.8 & -3.1 to 4.1 \\
\hline Interobserver & 1.4 & 2.4 & -3.4 to 6.2 \\
\hline \multicolumn{4}{|l|}{$\mathrm{h}(\mathrm{mm})$} \\
\hline Intraobserver (IC) & 0.1 & 0.3 & -0.4 to 0.7 \\
\hline Intraobserver (CR) & 0.1 & 0.2 & -0.4 to 0.6 \\
\hline Interobserver & 0.2 & 0.4 & -0.5 to 1.0 \\
\hline \multicolumn{4}{|l|}{$\alpha^{\prime}\left({ }^{\circ}\right)$} \\
\hline Intraobserver (IC) & 1.4 & 1.9 & -2.2 to 5.1 \\
\hline Intraobserver (CR) & 1.7 & 1.8 & -1.8 to 5.2 \\
\hline Interobserver & 1.8 & 2.1 & -2.3 to 6.0 \\
\hline \multicolumn{4}{|l|}{$\beta^{\prime}\left({ }^{\circ}\right)$} \\
\hline Intraobserver (IC) & 2.1 & 2.2 & -2.2 to 6.4 \\
\hline Intraobserver (CR) & 1.5 & 2.2 & -2.8 to 5.9 \\
\hline Interobserver & 1.4 & 3.5 & -5.5 to 8.3 \\
\hline
\end{tabular}

Intraobserver and interobserver relative differences were less than $5 \%$ for all parameters. The Bland-Altman method showed excellent agreement between intraobserver and interobserver measurements in both low and high values of echocardiographic parameters. Observers: observer 1, Irene Capecchi (IC); observer 2, Carlo Rostagno (CR); interobserver, (observer 1-observer 2). ESV, End-systolic volume; $L V E F$, left ventricular ejection fraction; ERO, effective regurgitant orifice (area); $h$, coaptation height; $\alpha^{\prime}$, anterior mitral leaflet tethering angle; $\beta^{\prime}$, posterior mitral leaflet tethering angle.

\section{Appendix 2. Tethering measurements}

The tethering angle of the AML $\left(\alpha^{\prime}\right)$ was measured as follows: $\alpha^{\prime}=\arcsin \left(\frac{B H}{B D}\right)$, where $\mathrm{BH}$ was the bending height between the annular line and the bending angle $(\gamma)$ created by tethering of intermediate or strut chordae in the body of the anterior leaflet, and $\mathrm{BD}$ was the bending distance between the bending point and the anterior annulus.

The AML excursion angle $\left(\alpha_{E X}\right)$ was obtained as follows: $\alpha_{E X}=\left[\left(\arcsin \frac{K}{A M L d}\right)-\left(\arcsin \frac{B H}{B D}\right)\right]$, where AMLd and $\mathrm{K}$ were the length of the anterior leaflet and the distance measured from the tip of the AML to the annular line, respectively, measured at the end of diastole.

Thus the anterior leaflet excursion $\left(\mathrm{AML}_{\mathrm{EX}}\right)$ was calculated as follows: $\mathrm{AML}_{\mathrm{EX}}=\mathrm{AML} * \sin \left[\left(\arcsin \frac{K}{\mathrm{AMLd}}\right)-\right.$ $\left.\left(\arcsin \frac{B H}{B D}\right)\right]$.

Similarly, the PML tethering angle $\left(\beta^{\prime}\right)$ was calculated as follows: $\beta^{\prime}=\arcsin \left(\frac{P M L s}{l^{\prime}}\right)$, where PMLs was the midsystolic PML and $l^{\prime}$ was the distance between the PML coaptation point and the annular line. 
The angle of PML excursion $\left(\beta_{E X}\right)$ and excursion distance $\left(P M L_{E X}\right)$ were calculated as follows: $\beta_{E X}=\left[\left(\arcsin \frac{l}{P M L d}\right)-\right.$ $\left.\left(\arcsin \frac{P M L S}{l^{\prime}}\right)\right] ; P M L_{E X}=\mathrm{PML} * \sin \left[\left(\arcsin \frac{l}{P M L}\right)-\left(\arcsin \frac{P M L S}{l^{\prime}}\right)\right]$, where PMLd and 1 were the length of the posterior leafletand the distance measured from the tip of the PML to the annular line, respectively, measured at the end of diastole.
The $\alpha^{\prime} / \beta^{\prime}$ ratio was calculated to verify the tethering symmetry.

The bending angle $(\gamma)$ was calculated as follows: $\gamma=90^{\circ}+\operatorname{arcos}\left(\frac{B H}{B D}\right)$. 